\title{
Characteristics of Eddy Current Distribution in Carbon Fiber Reinforced Polymer
}

\author{
Shaoni Jiao, Jian Li, Fei Du, Lei Sun, and Zhiwei Zeng \\ School of Aerospace Engineering, Xiamen University, Xiamen, Fujian 361005, China \\ Correspondence should be addressed to Zhiwei Zeng; zeng@xmu.edu.cn
}

Received 26 May 2016; Revised 22 July 2016; Accepted 31 July 2016

Academic Editor: Yinan Zhang

Copyright ( 2016 Shaoni Jiao et al. This is an open access article distributed under the Creative Commons Attribution License, which permits unrestricted use, distribution, and reproduction in any medium, provided the original work is properly cited.

\begin{abstract}
The paper studies the characteristics of eddy current (EC) distribution in carbon fiber reinforced polymer (CFRP) laminates so as to guide the research and operation of eddy current testing of CFRP. To this end, an electromagnetic field computation model of EC response to CFRP based on the finite element method is developed. Quantitative analysis of EC distribution in plies of unidirectional $\mathrm{CFRP}$ reveals that $\mathrm{EC}$ changes slowly along the fiber direction due to the strong electrical anisotropy of the material. Variation of EC in plies of multidirectional CFRP is fast in both directions. The attenuation of EC in the normal direction in unidirectional CFRP is faster than that in isotropic material due to faster diffusion of EC. In multidirectional CFRP, EC increases near the interfaces of plies having different fiber orientations. The simulation results are beneficial to optimizing sensor design and testing parameters, as well as damage detection and evaluation.
\end{abstract}

\section{Introduction}

Carbon fiber reinforced polymer (CFRP) has found wide applications, such as load-carrying structures in aircrafts, because of its outstanding performance [1-3]. Unfortunately, defects and damage are inevitable during its production and service in harsh environment, which deteriorates the performance of CFRP structures $[4,5]$. Therefore, nondestructive testing (NDT) is important for guaranteeing the quality and reliability of CFRP structures. Considering its electrically conducting property, CFRP structures can be examined by eddy current testing (ECT) technique [6-9]. Among various NDT methods [10-14], ECT is advantageous because of its easy operation, single-side detection, low requirement of surface preparation, and applicability in harsh environment, as well as other properties [15]. Hence ECT has taken an increasing interest in the detection and characterization of defects in CFRP recently [16-18].

Studying the characteristics of EC is beneficial to understanding field/flaw interaction, optimizing probe, and testing parameters, as well as guiding testing operations $[19,20]$. As CFRP is electrically anisotropic [16], the distribution of EC in CFRP must be different from that in isotropic material. It has been found that EC in CFRP induced by the excitation ac current in a circular coil flows in an elliptical shape [17, 18]. However, the phenomenon has not been quantitatively investigated. The paper studies the characteristics of EC distribution quantitatively and schematically. The research includes studying intraply distribution of EC and analyzing the attenuation of $\mathrm{EC}$ along the penetrating direction. In each case, characteristics of EC in both unidirectional CFRP and multidirectional CFRP are investigated.

The research is performed by numerical analysis. Section 2 presents briefly the simulation model and its validation. Section 3 studies the intraply distribution of EC. Section 4 investigates the attenuation of $\mathrm{EC}$ along the penetrating direction. Section 5 makes conclusive remarks.

\section{Numerical Simulation}

An air-core coil above a stratified CFRP laminate is modeled, as shown in Figure 1. Two Cartesian coordinate systems, namely, the global system and the local system, are used to describe the directions of fibers in the plies. The origin of the global system $(x, y, z)$ is at the center of the material surface. The local system $(l, t, n)$ is defined in each ply where $l$ is parallel to the fiber direction, $t$ is also intraply but 


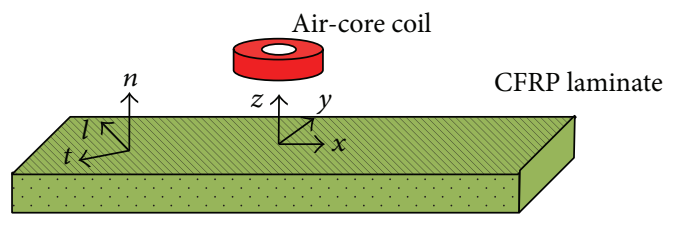

FIGURE 1: Geometrical model and coordinate systems.

perpendicular to $l$, and $n$ is along the normal vector of ply. The angle between the $l$-axis and the $x$-axis is the ply angle, denoted as $\theta$. The conductivity tensor of specific ply in global coordinate system can be formulated as [18]

$$
\begin{aligned}
& \overline{\bar{\sigma}} \\
& =\left[\begin{array}{ccc}
\sigma_{l} \cos ^{2}(\theta)+\sigma_{t} \sin ^{2}(\theta) & \frac{\sigma_{l}-\sigma_{t}}{2} \sin (2 \theta) & 0 \\
\frac{\sigma_{l}-\sigma_{t}}{2} \sin (2 \theta) & \sigma_{l} \sin ^{2}(\theta)+\sigma_{t} \cos ^{2}(\theta) & 0 \\
0 & 0 & \sigma_{n}
\end{array}\right],
\end{aligned}
$$

where $\sigma_{l}, \sigma_{t}$, and $\sigma_{n}$ are the conductivities along the $l, t$, and $n$ directions, respectively. $\operatorname{Diag}(10,000,100,100) \mathrm{S} / \mathrm{m}$ is a typical conductivity tensor of CFRP when $\theta=0$ [21].

The FE model for calculating EC response to CFRP structure is based on the $\mathbf{A}_{r}, V-\mathbf{A}_{r}$ formulation whose governing equations are [22]

$$
\begin{aligned}
& -\frac{1}{\mu_{0}} \nabla^{2} \mathbf{A}_{r}+j \omega \overline{\bar{\sigma}}\left(\mathbf{A}_{r}+\nabla v\right)=-\nabla \times \mathbf{H}_{s}-j \omega \overline{\bar{\sigma}} \mathbf{A}_{s} \\
& \nabla \cdot\left(-j \omega \overline{\bar{\sigma}} \mathbf{A}_{r}-j \omega \overline{\bar{\sigma}} \nabla v\right)=\nabla \cdot j \omega \overline{\bar{\sigma}} \mathbf{A}_{s} \quad\left(\Omega_{1}\right), \\
& -\frac{1}{\mu_{0}} \nabla^{2} \mathbf{A}_{r}=0 \quad\left(\Omega_{2}\right),
\end{aligned}
$$$$
\left(\Omega_{1}\right)
$$

where $\mathbf{A}_{r}$ and $V$ stand for the reduced magnetic vector potential and the electric scalar potential, respectively. $v=V /(j \omega)$ is introduced in (2) and (3). The CFRP laminate region $\left(\Omega_{1}\right)$ and the air region $\left(\Omega_{2}\right)$ including the current source constitute the whole solution domain. In (2)-(4), $\mathbf{A}_{s}$ and $\mathbf{H}_{s}$ are the magnetic vector potential and the magnetic field intensity in free space produced by the excitation current, respectively, $\mu_{0}$ is the permeability of free space, and $\omega$ is the angular frequency. The formulation has advantages of simple form and no requirement of meshing coil. For the details of the formulation and numerical implementation, please refer to [22]. After obtaining the solutions of the potentials, the coil impedance is calculated and the EC density $\mathbf{J}$ is formulated as

$$
\mathbf{J}=-j \omega \overline{\bar{\sigma}}(\mathbf{A}+\nabla v)
$$

where $\mathbf{A}$ is the sum of $\mathbf{A}_{s}$ and $\mathbf{A}_{r}$.

The model is validated by comparing simulation result and analytical solution of the change of coil's impedance due to $\mathrm{EC}$, denoted as $\Delta Z$, in a unidirectional CFRP $(\theta=0)$. The coil has inner diameter of $8 \mathrm{~mm}$, outer diameter of $12 \mathrm{~mm}$, height of $1 \mathrm{~mm}$, and lift-off of $1 \mathrm{~mm}$, and the number of

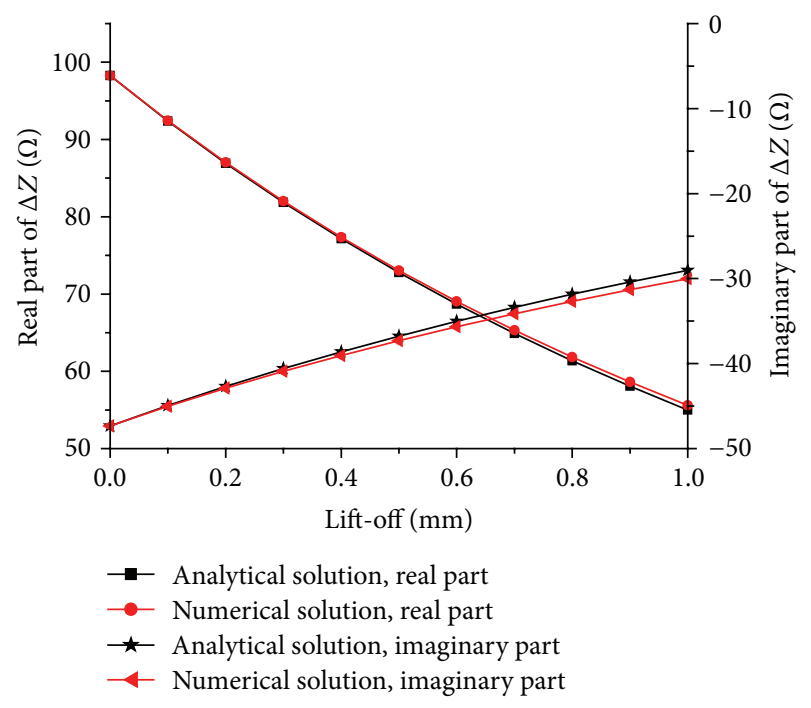

FIGURE 2: Change of coil impedance due to EC in CFRP as function of lift-off.

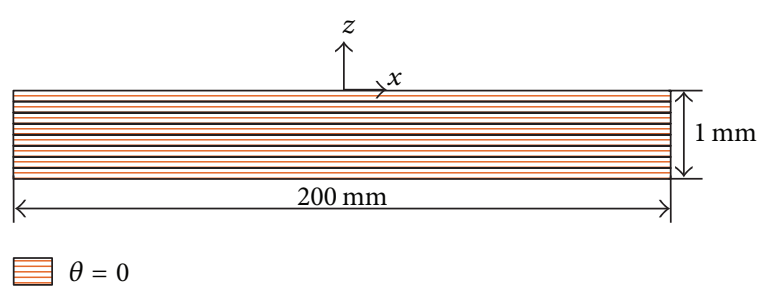

FIGURE 3: Geometrical model of anisotropic material.

turns is 50 . The carrying sinusoidal current has frequency of $10 \mathrm{MHz}$ and current density of $10^{7} \mathrm{~A} / \mathrm{m}^{2}$. The coil and the excitation current are used throughout the paper. The test material is a plate of $200 \mathrm{~mm} \times 200 \mathrm{~mm}$ area and $1 \mathrm{~mm}$ thickness. $\sigma_{l}, \sigma_{t}$, and $\sigma_{n}$ are chosen to be $10,000 \mathrm{~S} / \mathrm{m}, 100 \mathrm{~S} / \mathrm{m}$, and $100 \mathrm{~S} / \mathrm{m}$, respectively. The variation of $\Delta Z$ versus coil liftoff is shown in Figure 2. The numerical results agree well with the analytical solutions [23], which validates the effectiveness of the FE code.

\section{Intraply Variation of EC}

The distributions of EC in plies of unidirectional and multidirectional CFRP laminates are investigated in this section. All the CFRP laminates concerned here have eight plies and each ply is $0.125 \mathrm{~mm}$ thick.

3.1. Intraply Variation of EC in Unidirectional CFRP. To study the effect of electrical anisotropy on the distribution of EC, EC densities with three cases of conductivities are computed. The conductivities $\left(\sigma_{l}, \sigma_{t}, \sigma_{n}\right)$ are $(10,000,10,000,10,000) \mathrm{S} / \mathrm{m},(10,000,1,000,1,000) \mathrm{S} / \mathrm{m}$, and $(10,000,100,100) \mathrm{S} / \mathrm{m}$, respectively. The geometrical model of anisotropic material is shown in Figure 3. In these cases, the local coordinate system is position independent and is identical to the global coordinate system. Figure 4 shows the 


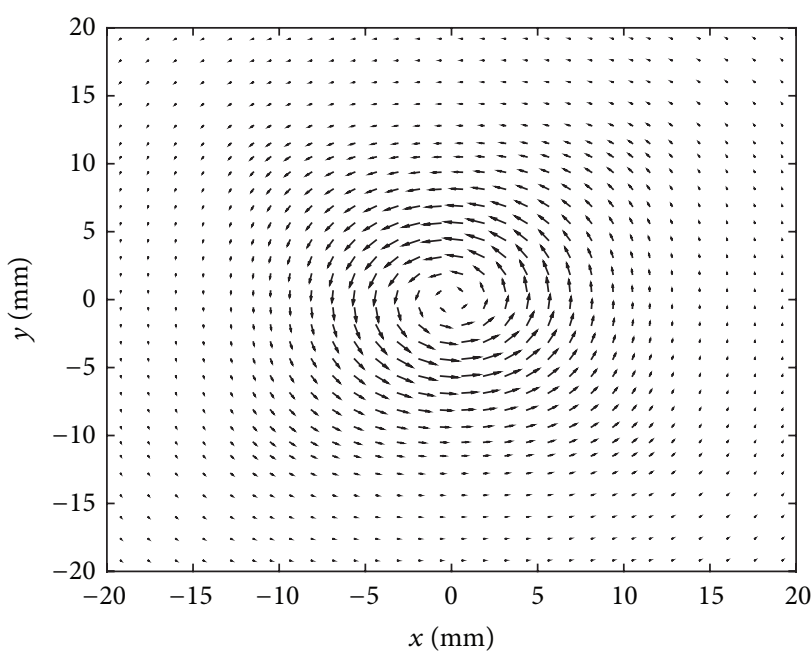

(a)

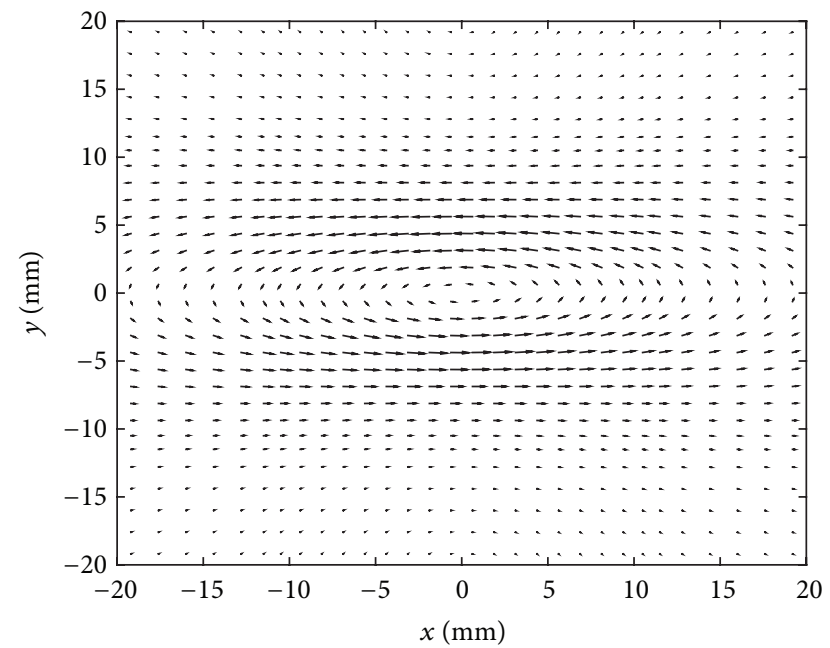

(b)

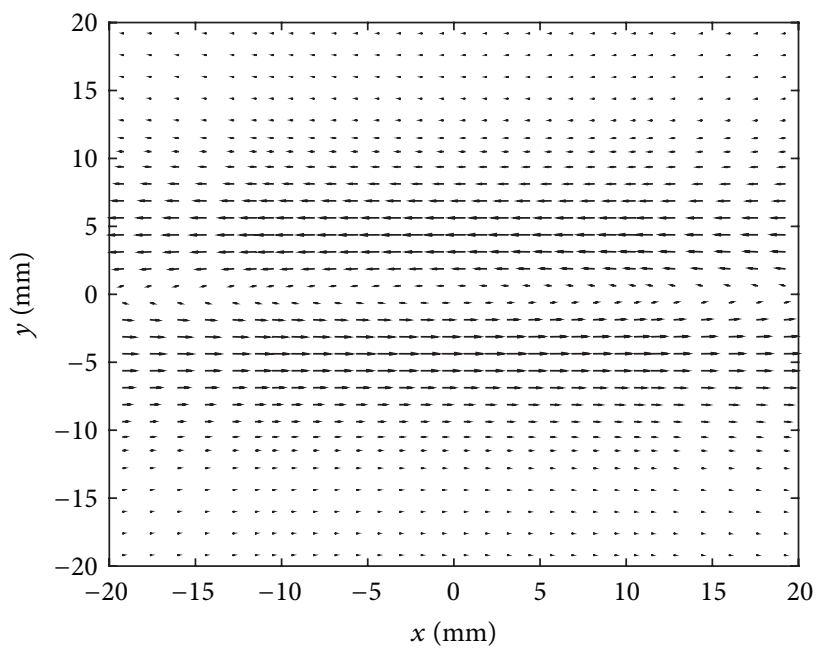

(c)

FIGURE 4: Distribution of EC (real part) in the top plies of the isotropic material and the unidirectional laminates with $\sigma_{l}=10,000 \mathrm{~S} / \mathrm{m}$ and (a) $\sigma_{t}=\sigma_{n}=10,000 \mathrm{~S} / \mathrm{m}$, (b) $\sigma_{t}=\sigma_{n}=1,000 \mathrm{~S} / \mathrm{m}$, and (c) $\sigma_{t}=\sigma_{n}=100 \mathrm{~S} / \mathrm{m}$.

real parts of EC densities in the $x-y$ plane of the top ply. We can easily see that EC flows in a circular shape in the isotropic material, whereas EC in each of the anisotropic laminates is mainly along the direction of maximum conductivity.

The EC density reaches maximum at the points of $x=0$, $y= \pm 5 \mathrm{~mm}$ regardless of the degree of anisotropy, as can be seen in Figure 4. Figures 5(a) and 5(b) show the EC densities along the $y$ direction with $x=0$ and along the $x$ direction with $y=5 \mathrm{~mm}$, respectively. It is indicated that the effect of electrical anisotropy on the rate of change of EC along the fiber direction is more significant than that along the direction perpendicular to the fibers. The variations of EC along the fiber direction in the anisotropic materials are much slower than that in the isotropic material. This phenomenon results in strong edge effect when the coil is near a side perpendicular to the fiber orientation and weak edge effect when the coil is close to a side parallel to the fiber orientation. Therefore, when doing scanning testing, moving probe in the direction transverse to fibers will have small and consistent influence of edge effect. In addition, from Figure 5 it is seen that, under the same excitation, more degree of anisotropy of material results in weaker EC response. This phenomenon indicates that the excitation current needs to be enlarged to induce strong enough EC when testing anisotropic material.

\subsection{Intraply Variation of EC in Multidirectional CFRP.} This subsection studies the intraply distribution of EC in multidirectional CFRP laminate. Four pieces of multidirectional CFRP laminates are used. The structures have laminate stacking sequences of $\left[0_{4} / 90_{4}\right],\left[0_{2} / 90_{2} / 0_{2} / 90_{2}\right]$, $\left[0_{2} /-45_{2} / 90_{2} / 45_{2}\right]$, and $[0 / 90 / 0 / 90 / 0 / 90 / 0 / 90]$. The subscript denotes the number of plies stacked successively with the same $\theta$. All the fibers in the top plies of the materials are oriented with $\theta=0$. Figure 6 illustrates the laminate structure of the material of $\left[0_{2} /-45_{2} / 90_{2} / 45_{2}\right]$. In the rest of the paper, 


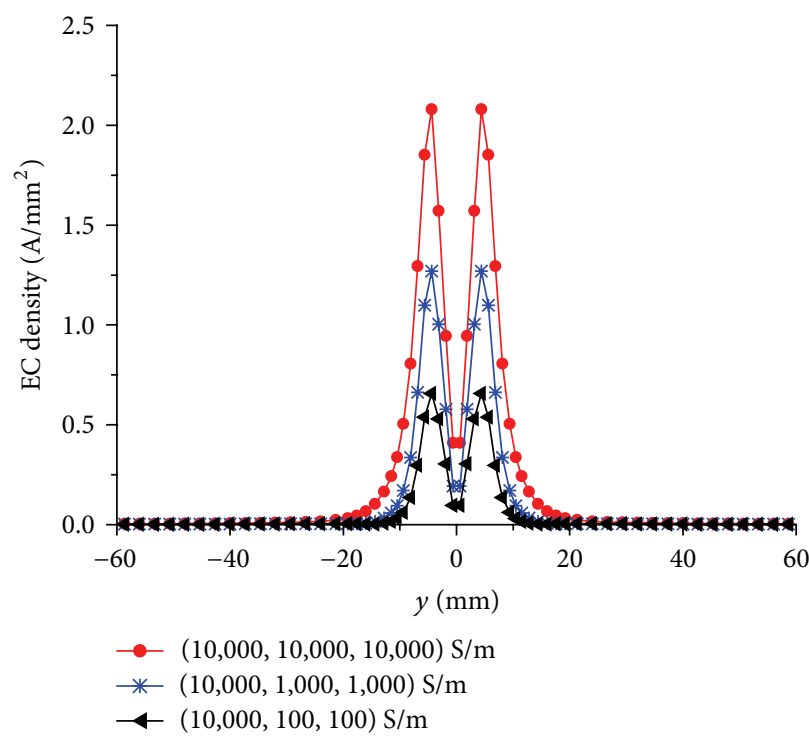

(a)

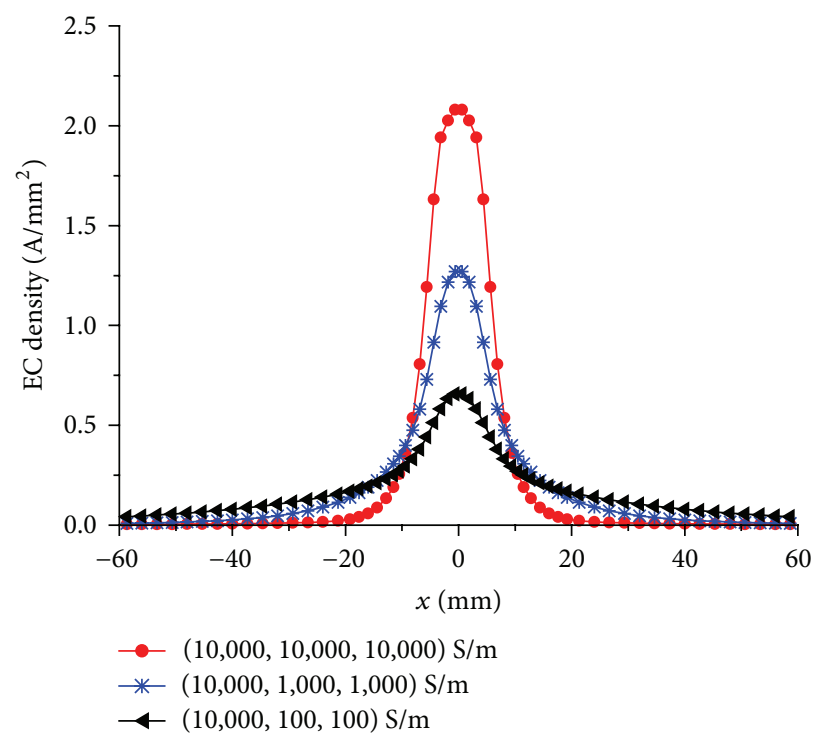

(b)

FIGURE 5: EC densities in the top plies of the isotropic material and the unidirectional laminates (a) along the $y$ direction with $x=0$ and (b) along the $x$ direction with $y=5 \mathrm{~mm}$.

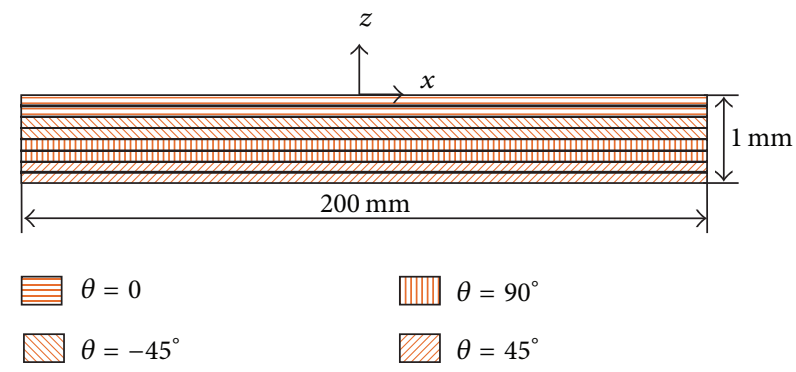

FIGURE 6: Geometrical model of the multidirectional CFRP laminate of $\left[0_{2} /-45_{2} / 90_{2} / 45_{2}\right]$.

$\sigma_{l}, \sigma_{t}$, and $\sigma_{n}$ of CFRP laminates are $10,000 \mathrm{~S} / \mathrm{m}, 100 \mathrm{~S} / \mathrm{m}$, and $100 \mathrm{~S} / \mathrm{m}$, respectively.

Figures $7(a)-7(d)$ show the distributions of EC in the 1st carbon ply $(\theta=0)$, the 3 rd ply $\left(\theta=-45^{\circ}\right)$, the 5th ply $\left(\theta=90^{\circ}\right)$, and the 7th ply $\left(\theta=45^{\circ}\right)$ of the laminate of $\left[0_{2} /-45_{2} / 90_{2} / 45_{2}\right]$, respectively. As is shown, EC flows mainly in the fiber orientation. Unlike the slow change of EC density along the fiber orientation in unidirectional CFRP, EC density in each ply of the multidirectional CFRP changes fast along fiber orientation. This is because EC in a fiber in a specific ply of multidirectional CFRP can change direction when it reaches the connecting point of the fiber and another fiber of different direction in an abutting ply.

Figures $8(\mathrm{a})$ and $8(\mathrm{~b})$ show the EC densities along the $y$ direction with $x=0$ and along the $x$ direction with $y=$ $5 \mathrm{~mm}$, respectively, in the top plies of the multidirectional laminates. The EC densities have similar rates of change along the $y$ direction, whereas the variations of EC along the $x$ direction in the multidirectional laminates are much faster than that in the unidirectional laminate. The more interfaces the multidirectional laminate has, the faster variation of $\mathrm{EC}$ along the $x$ direction is. As the distribution of EC in multidirectional laminate is compact in all directions, the influence of edge effect associated with multidirectional laminate is similar to that associated with isotropic material. Meanwhile the maximum values of EC density in the multidirectional laminates are larger than that in the unidirectional laminate, which can be explained by the extra EC paths as mentioned above and the reflection of EC on the interfaces of adjacent plies having fibers in different orientations in multidirectional laminates.

\section{Attenuation of EC in the Normal Direction}

In order to study the attenuation of EC in the normal direction, the thicknesses of the test materials are increased to $10 \mathrm{~mm}$ such that $\mathrm{EC}$ has become very small before reaching the bottom. Correspondingly each ply is of $1.25 \mathrm{~mm}$.

4.1. Skin Effect in Unidirectional CFRP. The FE model remains the same as the one used in Section 3.1 except that the thicknesses of the materials and of each ply are increased and the conductivities are changed. The conductivities $\left(\sigma_{l}, \sigma_{t}, \sigma_{n}\right)$ of the three materials are $(100,100,100) \mathrm{S} / \mathrm{m}$, $(10,000,10,000,10,000) \mathrm{S} / \mathrm{m}$, and $(10,000,100,100) \mathrm{S} / \mathrm{m}$, respectively. Figure 9 shows the amplitudes of EC densities as functions of depth with $x=0$ and $y=5 \mathrm{~mm}$ in the materials having various conductivity tensors. For each material, the EC densities are normalized by the value at the material surface. Obviously, the attenuation of EC in the anisotropic material is much faster than those in the isotropic materials; namely, the skin depth of EC in the anisotropic material is smaller than those in the isotropic materials, which is important for choosing testing parameters. 


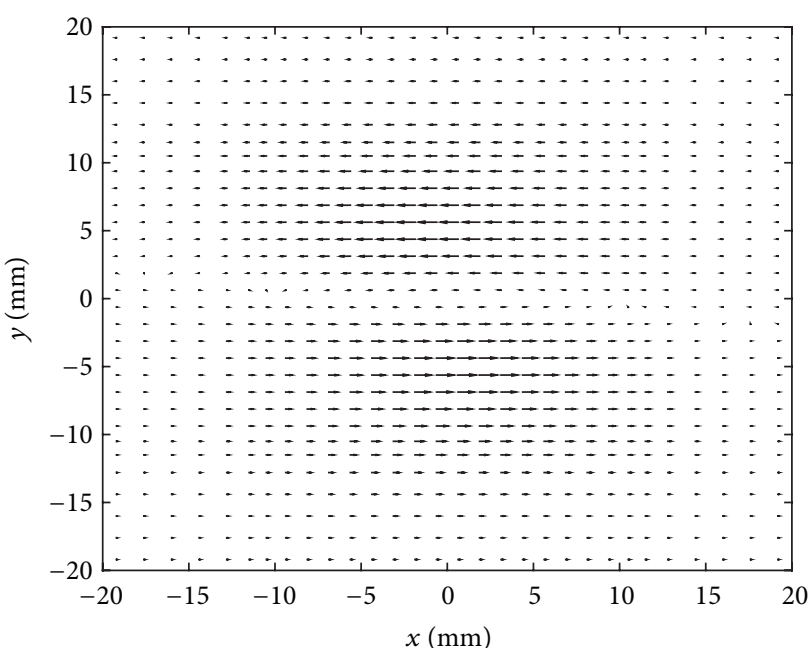

(a)

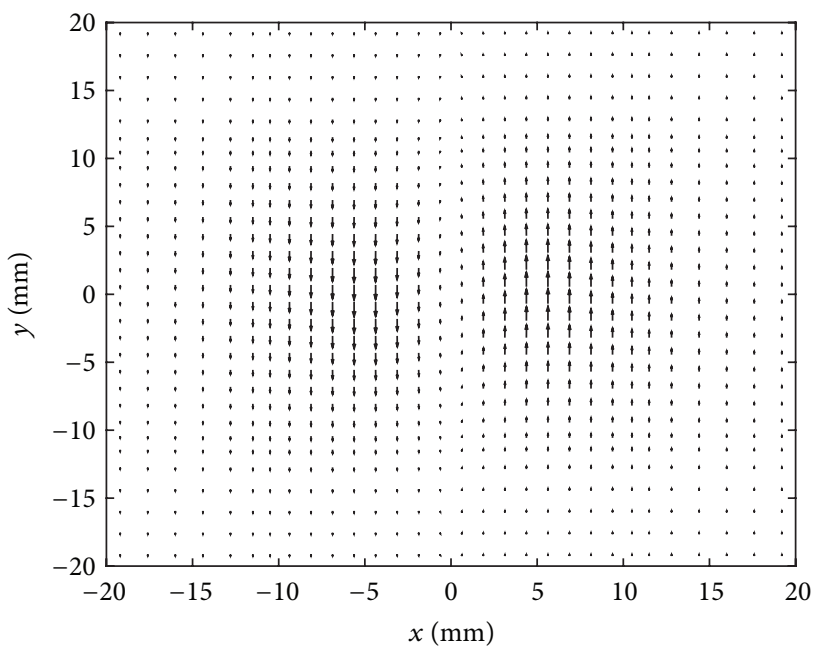

(c)

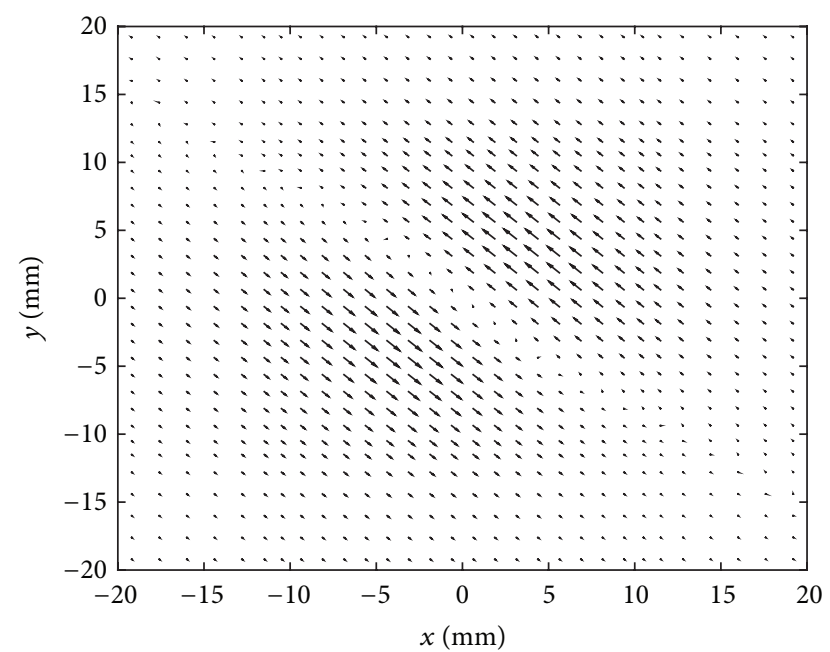

(b)

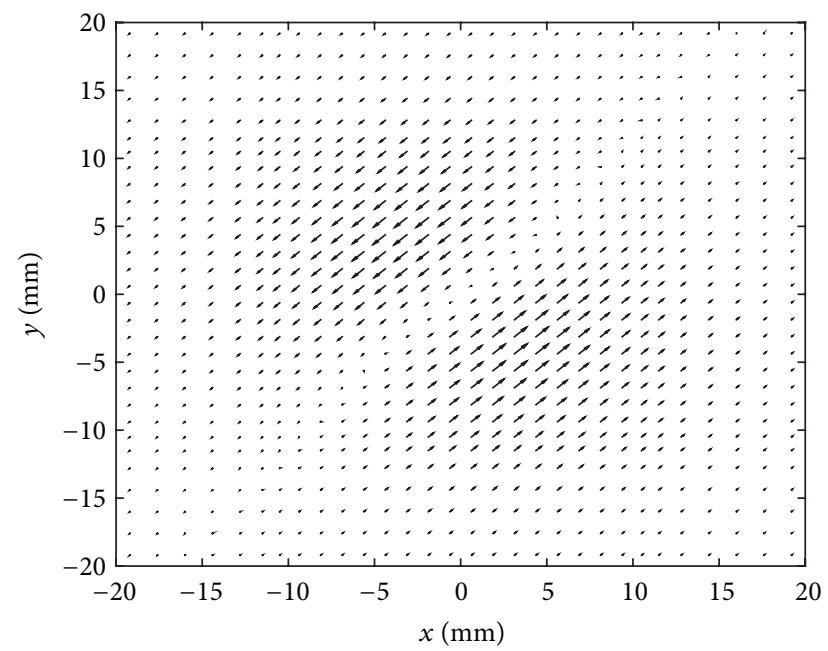

(d)

Figure 7: Distribution of EC (real part) in the multidirectional laminate of $\left[00_{2} /-45_{2} / 90_{2} / 45_{2}\right]$ in (a) the 1 st ply $(\theta=0)$, (b) the 3rd ply $\left(\theta=-45^{\circ}\right)$, (c) the 5th ply $\left(\theta=90^{\circ}\right)$, and (d) the 7th ply $\left(\theta=45^{\circ}\right)$.

To understand Figure 9, the diffusion and cancellation of EC are studied. Suppose an infinitely long single straight wire carrying ac current is placed parallel to the surface of a conductor, as shown in Figure 10(a). The spread of EC in a plane parallel to the surface becomes wider with increasing depth of the plane, as illustrated in Figure 10(b), which is referred to as diffusion of EC. At certain depth, define spread width of EC as the distance of the two points at which the values of EC are $1 / \sqrt{2}$ of the peak value. Let $L_{1}$ and $L_{2}$ be the spread widths of EC in the surface and at depth of $2 \mathrm{~mm}$, respectively. Their relative difference is

$$
\Delta L=\frac{L_{2}-L_{1}}{L_{1}}
$$

Larger $\Delta L$ means faster diffusion of EC. If another infinitely long straight wire is placed parallel to the previous wire with the same lift-off and the currents in the two wires are in opposite directions, then the ECs induced by the wires will have $180^{\circ}$ phase difference. Hence, their superposition results in the reduction of EC magnitude, which is referred to as cancellation of EC. Obviously faster diffusion of EC makes cancellation of EC more serious.

Simulation results show that the values of $\Delta L$ are $7.42 \%$, $15.93 \%$, and $26.28 \%$ for the samples having conductivities of $(100,100,100) \mathrm{S} / \mathrm{m},(10,000,10,000,10,000) \mathrm{S} / \mathrm{m}$, and $(10,000,100,100) \mathrm{S} / \mathrm{m}$, respectively. We can conclude that faster diffusion of EC in the anisotropic material makes cancellation of EC more serious, which makes attenuation of $\mathrm{EC}$ along the depth direction faster than those in the isotropic materials.

4.2. Skin Effect in Multidirectional CFRP. The multidirectional CFRP laminates used in Section 3.2 are utilized here for studying skin effect, with the thickness of each ply increased by 10 times. In a multidirectional laminate, the $(x, y)$ coordinates at which EC densities reach maximum of different 


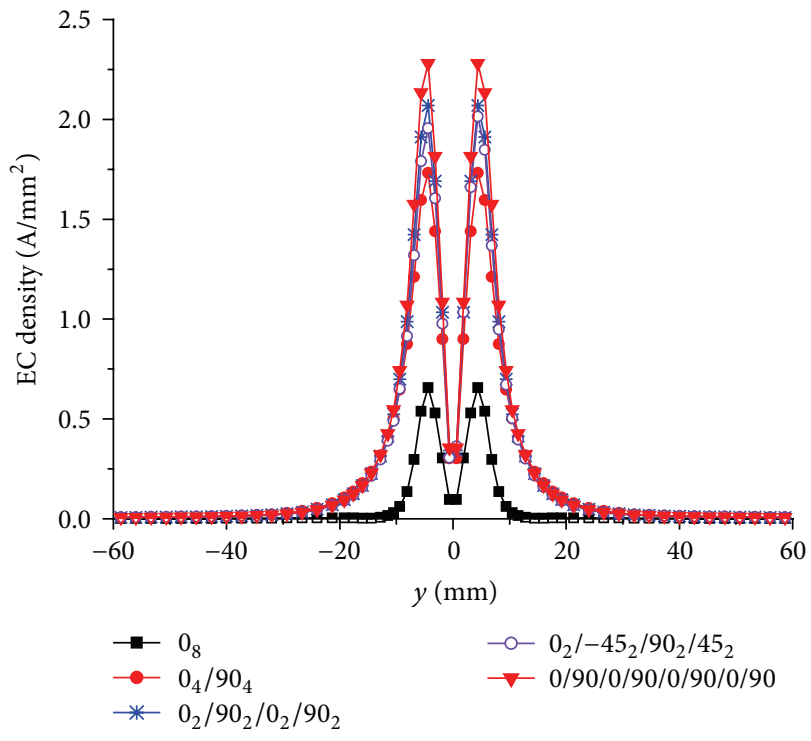

(a)

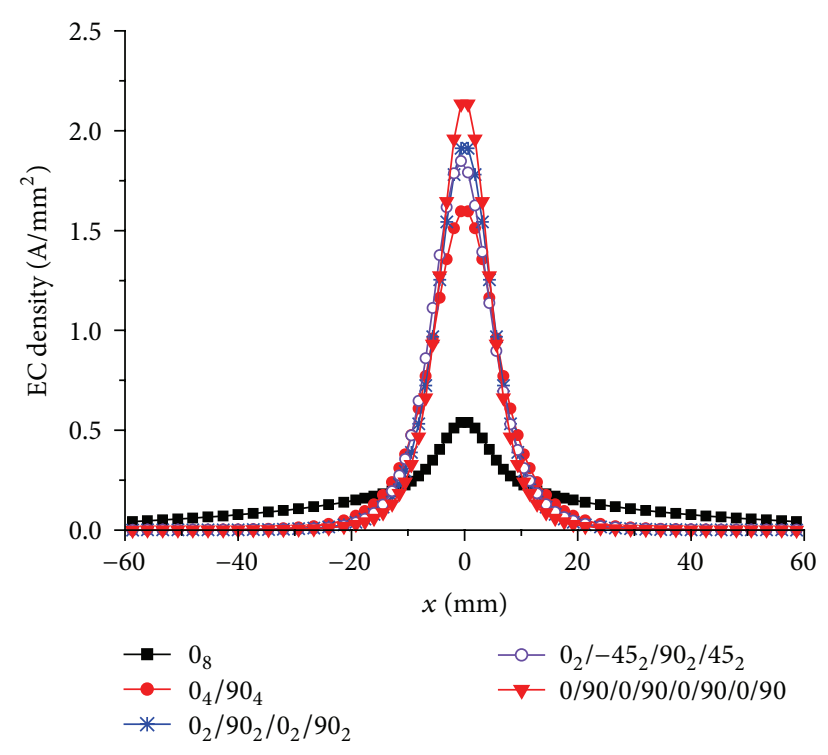

(b)

FIGURE 8: EC densities in the top plies $(\theta=0)$ of the multidirectional laminates: (a) along the $y$ direction with $x=0$ and (b) along the $x$ direction with $y=5 \mathrm{~mm}$.

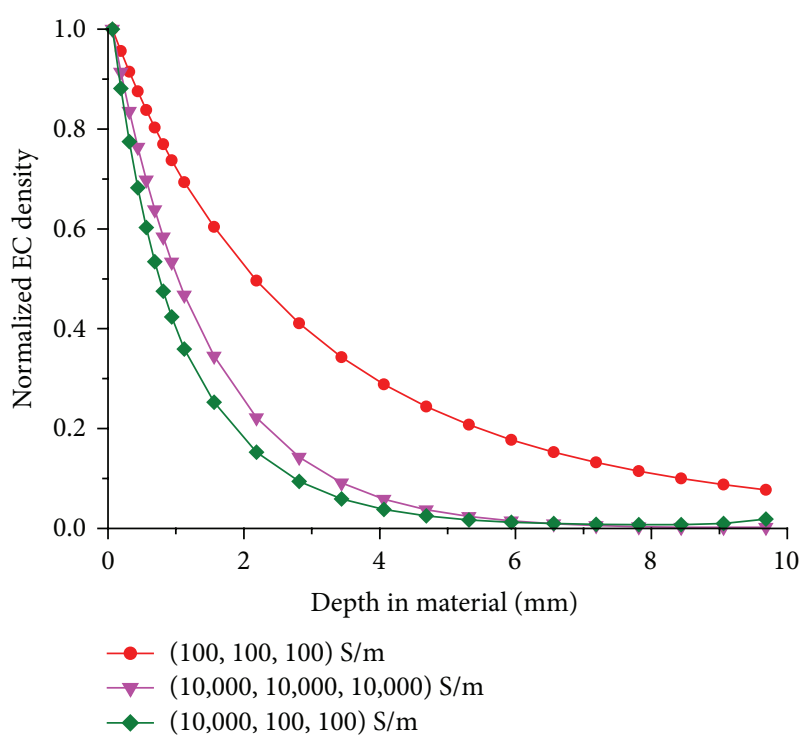

FIGURE 9: Attenuation of EC along the penetrating direction in isotropic and anisotropic materials.

plies may be different. For instance, the $(x, y)$ coordinates of maximum EC densities of the ply with $\theta=0$ and the ply with $\theta=90^{\circ}$ are $x=0, y= \pm 5 \mathrm{~mm}$ and $x= \pm 5 \mathrm{~mm}, y=0$, respectively. For each multidirectional laminate, we pick up the maximum EC densities in every layer of the FE mesh in the laminate and normalize them by the value in the top layer. Figure 11 shows the normalized EC densities as functions of depth of the four multidirectional laminates. The normalized EC density function of the unidirectional laminate is also shown for comparison. The points of calculating EC densities are inside the plies. The attenuation of EC in the $\left[0_{4} / 90_{4}\right]$ laminate is quite similar to that in the unidirectional laminate except that EC dramatically increases near the interface of the two adjacent plies having different fiber orientations, that is, the interface between the 4 th and the 5 th plies. The enhancement of EC near interface, for example, the interface between the 2nd and the $3 \mathrm{rd}$ plies, can also be easily seen in the attenuation curves of EC of the $\left[0_{2} / 90_{2} / 0_{2} / 90_{2}\right]$ and $\left[0_{2} /-45_{2} / 90_{2} / 45_{2}\right]$ laminates. In the $[0 / 90 / 0 / 90 / 0 / 90 / 0 / 90]$ laminate, there is only one peak in the attenuation curve of EC, because there are so many interfaces in the material and the increase of EC associated with an interface is largely affected by those associated with nearby interfaces.

It is concluded that the variation of EC in multidirectional laminate is different from that in unidirectional laminate due to extra EC paths and reflection of EC on interfaces and is affected by the stacking sequence of laminates. As the function of $\mathrm{EC}$ density versus depth is no longer monotonic, it is hard to define skin depth of EC in multidirectional CFRP.

\section{Conclusions}

Study of the characteristics of EC distribution is critical for designing probe and selecting testing parameters as well as damage detection and evaluation. The paper conducts quantitative analysis of EC distribution in unidirectional and multidirectional CFRP laminates.

Firstly, we study the intraply distribution of EC. In unidirectional CFRP, EC varies slowly along the fiber direction and changes fast in the orthogonal direction, which results in different levels of edge effect on different sides of the material. In multidirectional CFRP, the distribution of EC is compact in all directions. Therefore, the edge effect in this case is akin to that associated with isotropic material. 


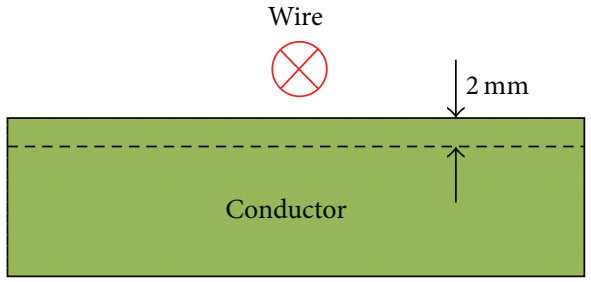

(a)

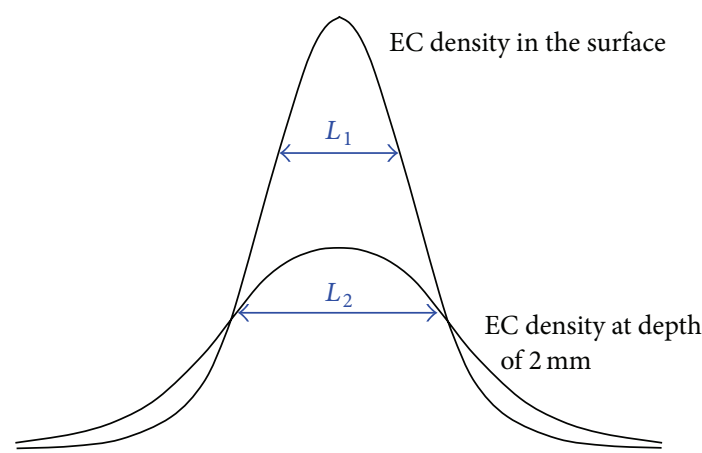

(b)

Figure 10: Illustration of diffusion of EC. (a) An infinitely long single straight wire carrying ac current placed above a conductor. (b) Distributions of EC in the conductor surface and at depth of $2 \mathrm{~mm}$.

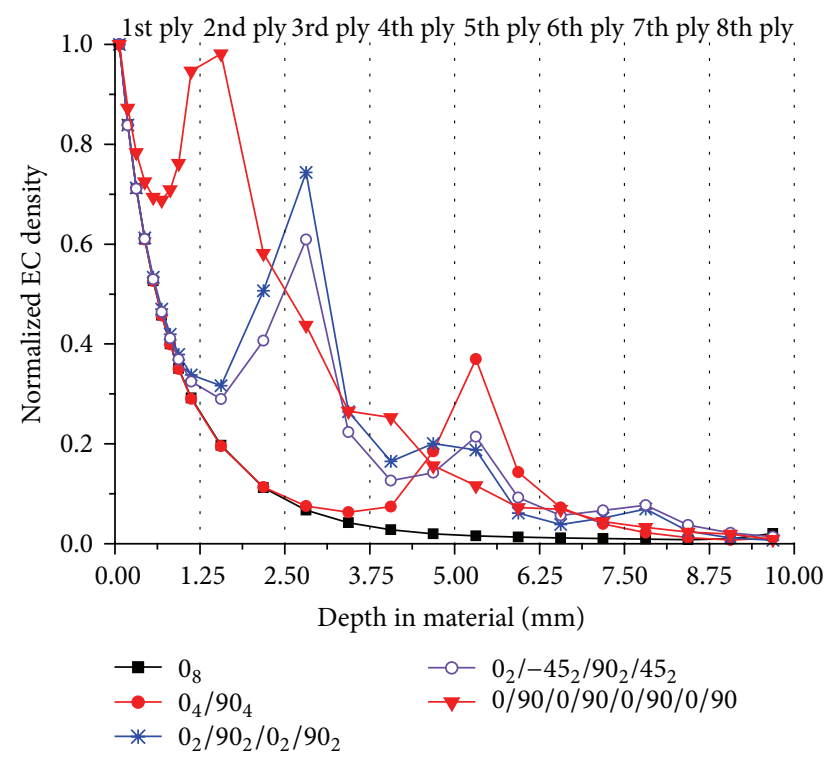

FIgURE 11: Attenuation of EC in the multidirectional CFRP laminates.

Then the attenuation of EC in the depth direction is analyzed. In unidirectional CFRP, EC attenuates faster than in isotropic material due to faster diffusion. In multidirectional CFRP, EC attenuates at similar speed as in the unidirectional CFRP except that it is enhanced near the interface of adjacent plies having different fiber orientations.

The characteristics of EC distribution in CFRP presented in the paper are obtained with working frequency of $10 \mathrm{MHz}$. When the frequency is $1 \mathrm{MHz}$, the characteristics of EC distribution are similar to those at $10 \mathrm{MHz}$.

\section{Competing Interests}

The authors declare that they have no competing interests.

\section{Acknowledgments}

This work is supported by the National Science Foundation of China under Grant 51277154 and the Research Fund for the Doctoral Program of Higher Education under Grant 20120121110026.

\section{References}

[1] H. A. Maples, S. Wakefield, P. Robinson, and A. Bismarck, "High performance carbon fibre reinforced epoxy composites with controllable stiffness," Composites Science and Technology, vol. 105, pp. 134-143, 2014.

[2] T. Bergmann, S. Heimbs, and M. Maier, "Mechanical properties and energy absorption capability of woven fabric composites under $\pm 45^{\circ}$ off-axis tension," Composite Structures, vol. 125, pp. 362-373, 2015.

[3] A. Todoroki, K. Yamada, Y. Mizutani, Y. Suzuki, and R. Matsuzaki, "Impact damage detection of a carbon-fibre-reinforcedpolymer plate employing self-sensing time-domain reflectometry," Composite Structures, vol. 130, pp. 174-179, 2015.

[4] H. K. Bui, G. Wasselynck, D. Trichet, B. Ramdane, G. Berthiau, and J. Fouladgar, "3-D modeling of thermo inductive non destructive testing method applied to multilayer composite," IEEE Transactions on Magnetics, vol. 49, no. 5, pp. 1949-1952, 2013.

[5] S. Zenia, L. B. Ayed, M. Nouari, and A. Delamézière, "Numerical prediction of the chip formation process and induced damage during the machining of carbon/epoxy composites," International Journal of Mechanical Sciences, vol. 90, pp. 89-101, 2015.

[6] S. Gäbler, H. Heuer, G. Heinrich, and R. Kupke, "Quantitatively analyzing dielectrical properties of resins and mapping permittivity variations in CFRP with high-frequency eddy current device technology," AIP Conference Proceedings, vol. 1650, pp. 336-344, 2015.

[7] J. Wu, D. Zhou, and J. Wang, "Surface crack detection for carbon fiber reinforced plastic materials using pulsed eddy current based on rectangular differential probe," Journal of Sensors, vol. 2014, Article ID 727269, 8 pages, 2014.

[8] X. Xu, M. Liu, Z. Zhang, and Y. Jia, "A novel high sensitivity sensor for remote field eddy current non-destructive testing 
based on orthogonal magnetic field," Sensors, vol. 14, no. 12, pp. 24098-24115, 2014.

[9] J. Cheng, H. Ji, J. Qiu, T. Takagi, T. Uchimoto, and N. Hu, "Role of interlaminar interface on bulk conductivity and electrical anisotropy of CFRP laminates measured by eddy current method," NDT \& E International, vol. 68, pp. 1-12, 2014.

[10] B. Akuthota, D. Hughes, R. Zoughi, J. Myers, and A. Nanni, "Near-field microwave detection of disbond in carbon fiber reinforced polymer composites used for strengthening cementbased structures and disbond repair verification," Journal of Materials in Civil Engineering, vol. 16, no. 6, pp. 540-546, 2004.

[11] S.-J. Yoon, D. Chen, S.-W. Han, N.-S. Choi, and K. Arakawa, "AE analysis of delamination crack propagation in carbon fiberreinforced polymer materials," Journal of Mechanical Science and Technology, vol. 29, no. 1, pp. 17-21, 2015.

[12] F. Sket, A. Enfedaque, C. Alton, C. González, J. M. MolinaAldareguia, and J. Llorca, "Automatic quantification of matrix cracking and fiber rotation by X-ray computed tomography in shear-deformed carbon fiber-reinforced laminates," Composites Science and Technology, vol. 90, pp. 129-138, 2014.

[13] L. Ma and M. Soleimani, "Hidden defect identification in carbon fibre reinforced polymer plates using magnetic induction tomography," Measurement Science and Technology, vol. 25, no. 5, Article ID 055404, 2014.

[14] Y. He, G. Tian, M. Pan, and D. Chen, "Impact evaluation in carbon fiber reinforced plastic (CFRP) laminates using eddy current pulsed thermography," Composite Structures, vol. 109, no. 1, pp. 1-7, 2014.

[15] Z. Liu, W. Li, F. Xue, J. Xia, B. Bu, and Z. Yi, "Electromagnetic tomography rail defect inspection," IEEE Xplore: IEEE Transactions on Magnetics, vol. 51, no. 10, Article ID 6201907, 1907.

[16] W. Yin, P. J. Withers, U. Sharma, and A. J. Peyton, "Noncontact characterization of carbon-fiber-reinforced plastics using multifrequency eddy current sensors," IEEE Transactions on Instrumentation and Measurement, vol. 58, no. 3, pp. 738-743, 2009.

[17] H. Menana and M. Féliachi, "An integro-differential model for 3-D eddy current computation in carbon fiber reinforced polymer composites," IEEE Transactions on Magnetics, vol. 47, no. 4, pp. 756-763, 2011.

[18] J. Cheng, H. Ji, J. Qiu, T. Takagi, T. Uchimoto, and N. Hu, "Novel electromagnetic modeling approach of carbon fiber-reinforced polymer laminate for calculation of eddy currents and eddy current testing signals," Journal of Composite Materials, vol. 49, no. 5, pp. 617-631, 2015.

[19] S. Bensaid, D. Trichet, and J. Fouladgar, "Optimal design of a rotating eddy-current probe-application to characterization of anisotropic conductive materials," IEEE Transactions on Magnetics, vol. 51, no. 3, Article ID 6200504, 2015.

[20] H. Menana and M. Féliachi, "Electromagnetic characterization of the CFRPs anisotropic conductivity: modeling and measurements," EPJ Applied Physics, vol. 53, no. 2, Article ID 21101, 2011.

[21] R. Grimberg, A. Savin, R. Steigmann, and A. Bruma, "Eddy current examination of carbon fibres in carbon-epoxy composites and Kevlar," International Journal of Materials and Product Technology, vol. 27, no. 3-4, pp. 221-228, 2006.

[22] L. Sun, Finite element analysis of eddy current testing of carbon fiber reinforced polymers [M.S. thesis], Xiamen University, 2014.

[23] S. K. Burke, "Eddy-current induction in a uniaxially anisotropic plate," Journal of Applied Physics, vol. 68, no. 7, pp. 3080-3090, 1990. 


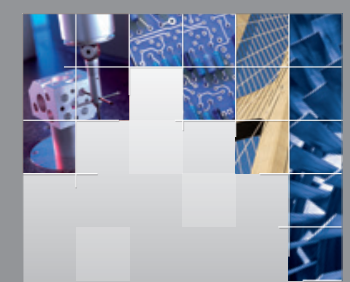

\section{Enfincering}
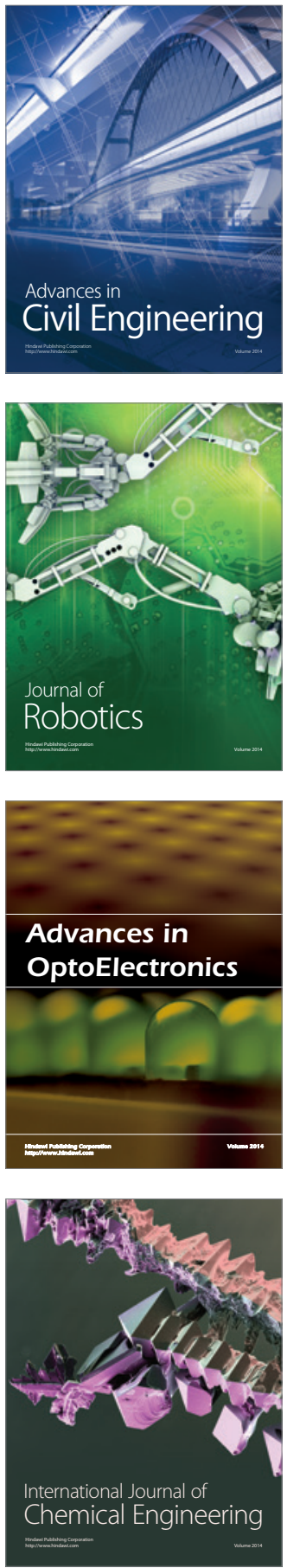

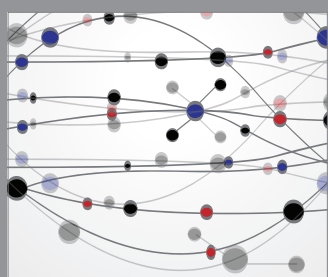

The Scientific World Journal

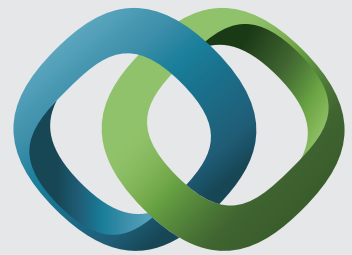

\section{Hindawi}

Submit your manuscripts at

http://www.hindawi.com
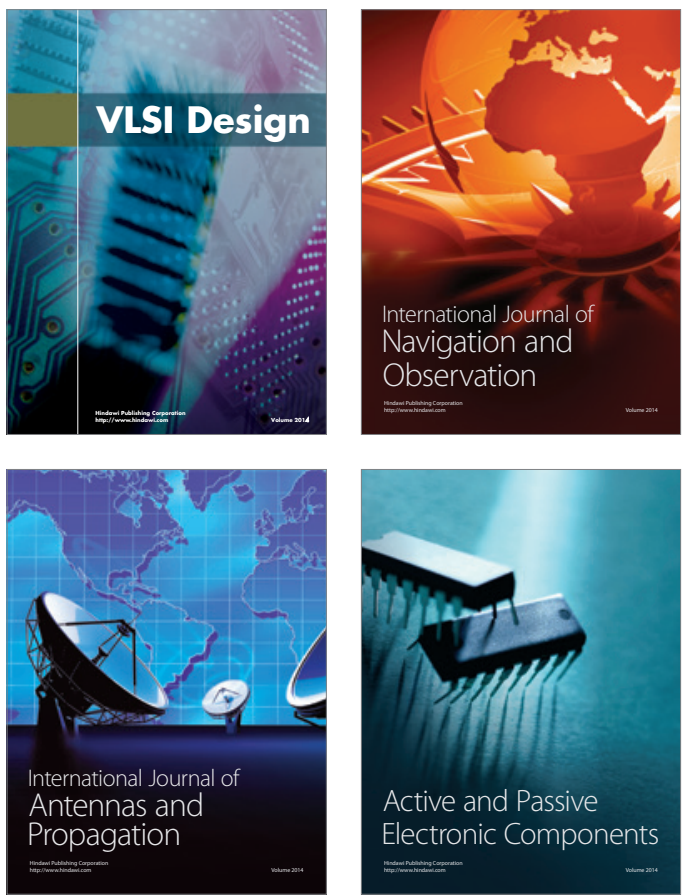
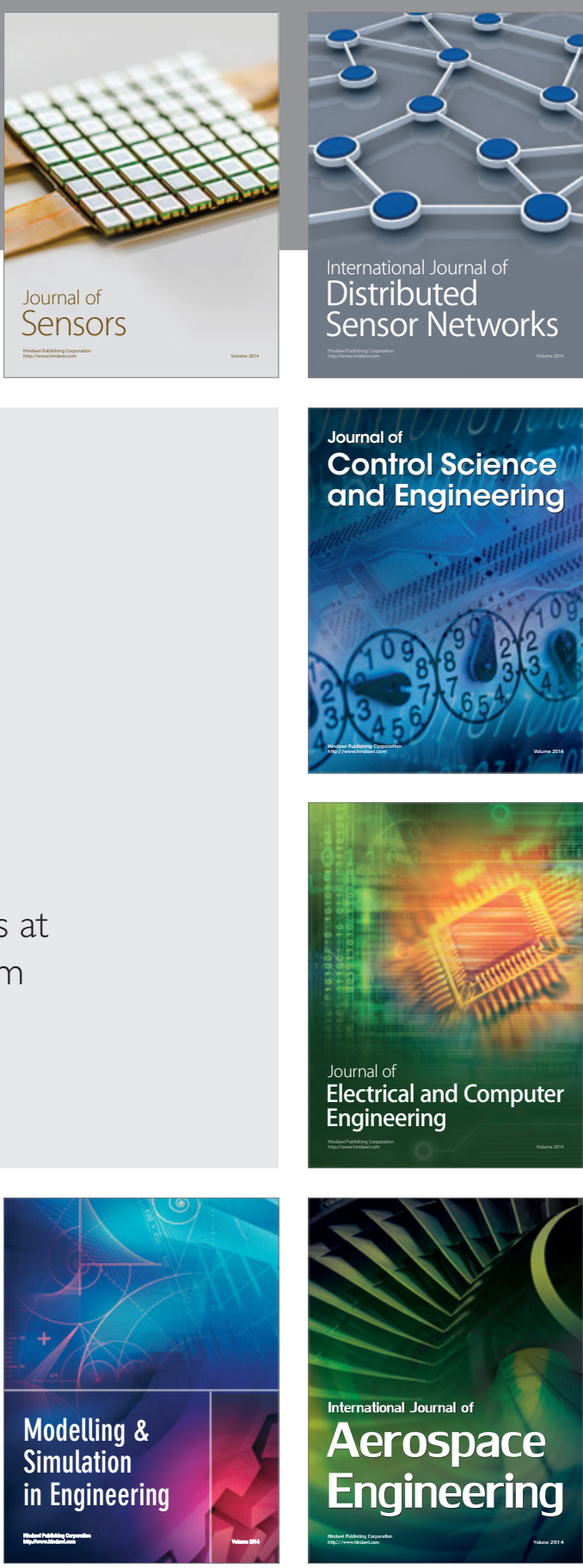

International Journal of

Distributed

Sensor Networks

Journal of

Control Science

and Engineering
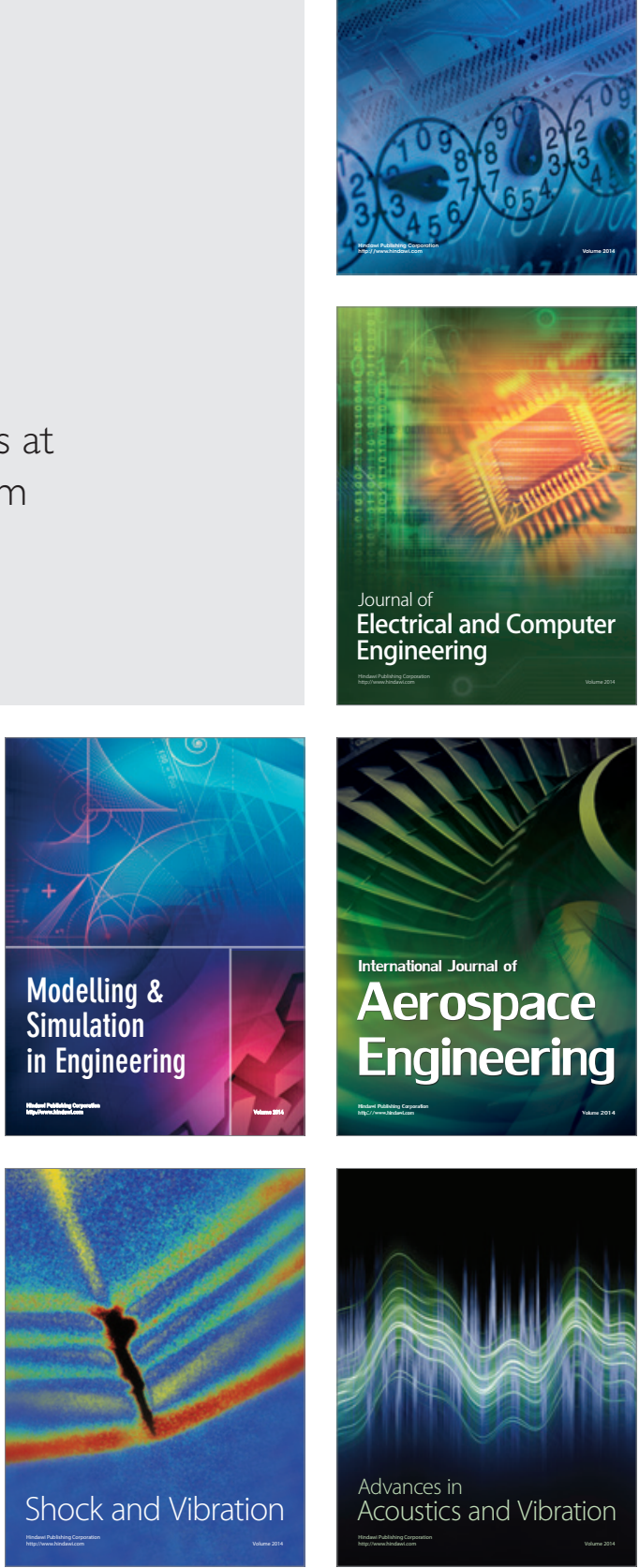